quintiles of the 2015 Index of Multiple Deprivation (IMD) as a measure of area-level socioeconomic deprivation. We used two measures of multimorbidity: a) basic multimorbidity: two or more chronic conditions; b) complex multimorbidity: at least three chronic conditions affecting at least three body systems. A list of 211 chronic conditions of interest, including long-term mental health conditions and chronic infections, was agreed by a multidisciplinary team. Using standard formulae, we calculated crude and age-sex standardised multimorbidity prevalence and incidence by geographical region. We used quasi-Poisson regression models to calculate risk ratios adjusted for year, sex, age, region, and IMD quintile. Analyses were conducted using R v4.0.4.

Results Our final sample consisted of 989,421 adults: 48.7\% male, with median age of 46 years (inter-quartile range 3362). The overall crude prevalence of multimorbidity in England was $43.7 \%$ for basic, and $25.2 \%$ for complex multimorbidity over the 16-year study period. London had the lowest crude prevalence of both multimorbidity types (basic: $35.4 \%$; complex: 18.3\%), whilst the North East had the highest (basic: 48.6\%; complex: 29.6\%). In age-sex standardised results, prevalence was still highest in the North East, with London and the South East having the lowest prevalence. Similar regional inequalities were found in the incidence of both multimorbidity types. Compared to London, the North East had higher multimorbidity prevalence in risk ratios adjusted for socioeconomic deprivation and demographic factors (basic multimorbidity: 1.18 (95\% confidence interval 1.16, 1.19); complex multimorbidity: 1.26 (95\% confidence interval 1.24, 1.29). Conclusion There are regional inequalities in multimorbidity within England with higher burden in the North, compared to London and the South. These inequalities remained after adjusting for age and socioeconomic deprivation. Strategies aimed at addressing the social determinants of health are needed to reduce future burden on health and social care systems, particularly in the North of England.

\section{P62 EDUCATIONAL INEQUALITIES IN STATIN TREATMENT: CROSS-SECTIONAL ANALYSIS OF UK BIOBANK}

\begin{abstract}
1,2Alice Carter* , 3,4,5,6,7 Dipender Gill, ${ }^{2,8}$ Richard Morris, ${ }^{1,2,9}$ George Davey Smith, ${ }^{2,9}$ Amy Taylor, ${ }^{1,2,1}$ Neil Davies, ${ }^{1,2}$ Laura Howe. 'MRC Integrative Epidemiology Unit, University of Bristol, Bristol, UK; ${ }^{2}$ Population Health Sciences, University of Bristol, Bristol, UK; ${ }^{3}$ Department of Epidemiology and Biostatistics, Imperial College London, London, UK; ${ }^{4}$ Centre for Pharmacology and Therapeutics, Imperial College London, London, UK; ${ }^{5}$ Clinical Pharmacology and Therapeutics Section, St George's, University of London, London, UK; ${ }^{6}$ Clinical Pharmacology Group, St George's, University of London, London, UK; ${ }^{7}$ Centre for Academic Primary Care, University of Bristol, Bristol, UK; ${ }^{8}$ NIHR Bristol Biomedical Research Centre, University of Bristol, Bristol, UK; ${ }^{9}$ K.G. Jebsen Center for Genetic Epidemiology, Norwegian University of Science and Technology, Trondheim, Norway
\end{abstract}

10.1136/jech-2021-SSMabstracts. 150

Background Despite reductions in the rates of cardiovascular disease in high income countries, individuals who are the most socioeconomically deprived remain at the highest risk of disease. Although intermediate lifestyle and behavioural risk factors explain some of this, much of the effect remains unexplained. It is not known whether differences in risk adjusted use of statins between educational groups may contribute to these inequalities.

Methods Using data from a large prospective cohort study, UK Biobank, we calculated a QRISK3 cardiovascular risk score for 472097 eligible participants with complete data on self- reported educational attainment and statin use (55\% female; mean age, 56). We used logistic regression to explore the association between i) QRISK3 score and ii) educational attainment on self-report statin use. We then stratified the association between QRISK3 score, and statin use by educational attainment to test for interactions. We then replicated analyses using QRISK or QRISK2 scores recorded in primary care data and statin prescriptions recorded in primary care prescription records.

Results There was evidence of an interaction between QRISK3 scores and education. For an equivalent QRISK3 score, more educated individuals were more likely to report taking statins. In women with 7 years of schooling, a one unit increase in QRISK3 score was associated with a 7\% higher odds of statin use (odds ratio (OR) 1.07, 95\% CI 1.07, 1.07). In women with 20 years of schooling, a one unit increase in QRISK3 score was associated with an $14 \%$ higher odds of statin use (OR 1.14, 95\% CI 1.14, 1.15). Comparable ORs in men were $1.04(95 \%$ CI $1.04,1.05)$ for 7 years of schooling and 1.08 (95\% CI 1.08, 1.08) for 20 years of schooling. These inequalities were also present in analyses using primary care data.

Conclusion For the same level of cardiovascular risk, individuals with lower educational attainment are less likely to receive statins, likely contributing to cardiovascular inequalities. The mechanisms leading to these differences are unknown, but both health seeking behaviours and clinical factors may contribute.

\section{P63 USING DATA ON FATHERS/PARTNERS TO STUDY PRENATAL PARENTAL EXPOSURES AND CHILD HEALTH: CHALLENGES INTRODUCED BY MISSING DATA AND SELECTION BIAS}

Kayleigh Easey*, Gemma Sharp. MRC Integrative Epidemiology Unit, University of Bristol, Bristol, UK

\subsection{6/jech-2021-SSMabstracts. 151}

Background There are challenges in studying the effects of partner exposures around pregnancy on child health. We explored potential sources of bias in the effects of parental prenatal health behaviours on child health, to describe and quantify some of these challenges, as well as suggest ways in which they might be mitigated.

Methods First, we characterised the availability of data on partner and mother health behaviours in the prenatal period from three UK cohort studies: the Avon Longitudinal Study of Parents and Children (ALSPAC), Born in Bradford (BiB), and the Millennium Cohort Study (MCS). Second, we assessed the potential for sample selection in these cohorts by comparing characteristics of families where the partner did and did not participate. Third, using parental smoking during pregnancy and child birthweight as an example, we ran simulation studies of several DAGs to explore the extent that missing partner data and selection can affect estimates. We then explored the 'real life' impact of partner sample selection on estimates of maternal effect.

Results In all cohorts, data on partner prenatal health behaviours was less detailed and collected less frequently than maternal prenatal health behaviours. Partners participated in ALSPAC and MCS for the majority of pregnancies. Of 14,472 pregnancies in ALSPAC, and 18,241 pregnancies in MCS, 
$12,997(78 \%)$ and $13,145(71 \%)$ had a cohort participating partner, respectively. However, partner participation was much lower in BiB: 3131/11,538 pregnancies (27\%) had a cohort participating partner. Consistently across all cohorts, in pregnancies with cohort participating partners, mothers were more likely to be living with their partner before birth of the child, be white and have a university degree. They were consistently less likely to have no qualifications and their babies were less likely to be born preterm or have a low birthweight. We saw relatively stable effect estimates within cohorts for associations between maternal smoking and offspring birthweight regardless of whether we used the full sample, the sample where fathers participated (selected either through stratification or adjusting for partner smoking), or the sample where fathers did not participate.

Conclusion Overall, these results show partner selection is unlikely to cause strong selection bias in estimates of maternal effects. This suggests that, although inclusion of partner data with high levels of non-random missingness has the potential to introduce selection bias, in practice, the biasing effect appears to be small. This also has implications for studies of partner effects in their own right.

\section{P64 HOW DO CHANGES IN INDIVIDUAL OR HOUSEHOLD INCOME IMPACT ON MENTAL HEALTH FOR WORKING- AGE ADULTS? A SYSTEMATIC REVIEW}

Rachel M Thomson*, Erik Igelström, Amrit Kaur Purba, Michal Shimonovich, Anna Pearce, Alastair Leyland, Hilary Thomson, S Vittal Katikireddi. MRC/CSO Social and Public Health Sciences Unit, University of Glasgow, Glasgow, UK

\subsection{6/jech-2021-SSMabstracts. 152}

Background Lower incomes are associated with poorer mental health $(\mathrm{MH})$ and wellbeing, but the extent to which income has a causal effect (rather than reflecting reverse causation or confounding factors such as education) is debated. We synthesised evidence from studies measuring the impact of changes in individual or household incomes on $\mathrm{MH}$ and wellbeing for working-age adults (16-64 years).

Methods We searched MEDLINE, Embase, Web of Science, PsycINFO, ASSIA, EconLit and RePEc for randomised controlled trials (RCTs) and quantitative non-randomised studies (NRSs) - PROSPERO registration CRD42020168379. We included studies measuring effects of income change on any $\mathrm{MH}$ or wellbeing outcome. Screening and risk of bias (RoB) assessment were completed independently by two reviewers, using ROBINS-I for NRSs and RoB-2 for RCTs. As per Cochrane guidance, we conducted narrative synthesis based on direction of effects (benefit vs harm) for relevant datapoint(s) within each included study, and compared findings by RoB. Meta-analysis is in progress.

Results Of 16,521 hits screened, 17 RCTs and 118 NRSs (67.0\% longitudinal) were included. Most studies were from high-income settings (71.9\%), with $26.7 \%$ from USA. RoB was high: 102 studies (75.6\%) were rated serious/critical, with confounding being the highest-rated RoB domain in $81.1 \%$ of these studies. Where known $(53.7 \%$ of studies), the most common income sources studied were cash transfers (20.2\%), natural disasters/welfare policy changes $(7.5 \%)$ and lottery wins (5.2\%). For mental health, $80.4 \%$ of 112 datapoints reported beneficial effects of income (95\% CI 73.0-87.7\%, sign test $\mathrm{p}<0.001)$. For wellbeing outcomes $86.5 \%$ of 74 datapoints reported a beneficial effect (95\%CI 78.7-94.3\%, $\mathrm{p}<0.001)$. However, effect sizes were small: for low/moderate RoB datapoints, binary exposures (i.e. increases/decreases in income of any amount) were associated with median 0.089 standard deviation (SD) improvement/worsening in outcome respectively (IQR $0.162, \mathrm{n}=24$ ); a $1 \mathrm{SD}$ increase/decrease in continuous exposures was associated with median $0.008 \mathrm{SD}$ improvement/worsening (IQR 0.276, $n=4$ ). Studies with low/ moderate RoB were slightly less likely to report beneficial effects compared with serious/critical RoB studies $(77.6 \%$ vs $84.7 \%, \mathrm{p}=0.257)$.

Conclusion Income increases are linked to improved $\mathrm{MH}$ and wellbeing, but on preliminary synthesis effect sizes appear small in the most robust studies. Income provision alone may not be adequate to improve mental health - given that more generous welfare policies are known to be linked with better population $\mathrm{MH}$, it is possible other elements such as conditionality or financial security are also important.

\section{P65 POTENTIAL ALCOHOL USE DISORDER (AUD) AMONG MEN WHO HAVE SEX WITH MEN (MSM) IN IRELAND - FINDINGS FROM THE EUROPEAN MSM INTERNET SURVEY (EMIS) 2017}

\begin{abstract}
${ }^{1,2}{ }^{2}$ Fionn P Daly* ${ }^{3}$ Kate O'Donnell, ${ }^{2,4}$ Martin P Davoren, ${ }^{5}$ Chris Noone, ${ }^{6}$ Peter Weatherburn, ${ }^{7}$ Mick Quinlan, ${ }^{7}$ Bill Foley, ${ }^{3}$ Derval Igoe, ${ }^{2,8}$ Peter M Barrett. ${ }^{1}$ School of Medicine and Health, University College Cork, Cork, Ireland; ${ }^{2}$ School of Public Health, University College Cork, Cork, Ireland; ${ }^{3}$ Health Protection Surveillance Centre, Health Service Executive, Dublin, Ireland; ${ }^{4}$ Sexual Health Centre, Ireland, Cork, Ireland; ${ }^{5}$ School of Psychology, National University of Ireland Galway, Galway, Ireland; ${ }^{6}$ Sigma Research, London School of Hygiene and Tropical Medicine, London, UK; ${ }^{7}$ Gay Health Network, Ireland, Dublin, Ireland; ${ }^{8}$ Department of Public Health HSE-South, St. Finbarr's Hospital, Cork, Ireland
\end{abstract}

\subsection{6/jech-2021-SSMabstracts. 153}

Background Alcohol consumption is a major public health concern in Ireland. Alcohol use disorder (AUD) disproportionately affects men who have sex with men (MSM). However, little is known about the prevalence of AUD in this group in Ireland specifically, and the characteristics of MSM who may struggle with this.

Methods The European MSM Internet Survey 2017 was an online, self-completed, anonymous questionnaire among MSM in Ireland. Standardised questions were used to explore a variety of topics. The validated CAGE-4 questionnaire was used to screen for potential AUD, defined as a CAGE-4 score of $\geq 2$ out of 4 . Multivariable-adjusted logistic regression analysis was used to identify factors associated with potential AUD.

Results In total, 1,793 MSM met inclusion criteria, and $31 \%$ screened positive for AUD. We observed higher odds of possible AUD among MSM who were bisexual (vs. gay/ homosexual) (aOR $1.4895 \% \mathrm{CI} 1.01-2.18$ ), native to Ireland (vs. non-native) (aOR 1.49 95\% CI 1.12-1.96), unemployed (vs. employed) (aOR 1.80 95\%CI 1.02-3.16), had used illicit drugs in the previous year (vs. none) (cannabis only, aOR 1.74 95\%CI 1.14-2.63) (other illicit drugs, aOR 2.28 95\%CI 1.67-3.09), reported anxiety/depression (vs. none) (aOR 1.73 95\%CI 1.12-2.66), and MSM who experienced homophobic abuse (vs. never) (aOR 1.55 95\%CI 1.09-2.22). Student MSM were less likely to screen positive for AUD (vs. employed) (aOR 0.65 95\%CI $0.46-$ $0.93)$.

Conclusion The prevalence of AUD appears to be higher in the MSM population compared to the general male 